Commun. Math. Phys. 183, $119-143$ (1997)

Communications in

Mathematical

Physics

(C) Springer-Verlag 1997

\title{
The Behavior of the Weyl Function in the Zero-Dispersion KdV Limit
}

\author{
Nicholas M. Ercolani, C. David Levermore, Taiyan Zhang * \\ Department of Mathematics, University of Arizona, Tucson, AZ 85721, USA. \\ E-mail: ercolani@math.arizona.edu, lvrmr@math.arizona.edu, taizhang@math.arizona.edu
}

Received: 13 June 1994/Accepted: 20 May 1996

Dedicated to Peter Lax on his $70^{\text {th }}$ birthday

\begin{abstract}
The moment formulas that globally characterize the zero-dispersion limit of the Korteweg-deVries (KdV) equation are known to be expressed in terms of the solution of a maximization problem. Here we establish a direct relation between this maximizer and the zero-dispersion limit of the logarithm of the Jost functions associated with the inverse spectral transform. All the $\mathrm{KdV}$ conserved densities are encoded in the spatial derivative of these functions, known as Weyl functions. We show the Weyl functions are densities of measures that converge in the weak sense to a limiting measure. This limiting measure encodes all of the weak limits of the KdV conserved densities. Moreover, we establish the weak limit of spectral measures associated with the Dirichlet problem.
\end{abstract}

\section{Introduction}

This paper presents a global interpretation of the moment formulas that characterize the zero-dispersion limit of the Korteweg-deVries equation (KdV) and which were first described in $[18,19]$. The problem is to determine the limit

$$
\lim _{\varepsilon \rightarrow 0} u^{\varepsilon}(x, t),
$$

where $u^{\varepsilon}$ solves the initial-value problem

$$
\begin{gathered}
\partial_{t} u^{\varepsilon}-6 u^{\varepsilon} \partial_{x} u^{\varepsilon}+\varepsilon^{2} \partial_{x x x} u^{\varepsilon}=0, \\
u^{\varepsilon}(x, 0)=v(x),
\end{gathered}
$$

for $v$ of scattering class and independent of $\varepsilon$. More generally, the limit (1.1) can be considered for $u^{\varepsilon}(x, t)$, the simultaneous solution of the whole $\mathrm{KdV}$ hierarchy of commuting flows with initial data given by $(1.2 b)$. Here $t=\left(t_{0}, t_{1} \cdots\right)$ denotes an infinite vector of times corresponding to the $\mathrm{KdV}$ flows where all but finitely many of the $t_{m}$ are zero. USA.

* Current address: Department of Mathematics, University of New Orleans, New Orleans, LA 70148, 
Basing their analysis on the WKB asymptotics of the associated inverse spectral problem for the Schrödinger operator

$$
-\varepsilon^{2} \partial_{x x} f^{\varepsilon}+u^{\varepsilon}(x, \mathbf{t}) f^{\varepsilon}=k^{2} f^{\varepsilon},
$$

Lax and Levermore $[18,19]$ established the limit (1.1) for the case of smooth nonpositive initial data $v(x)$ that vanishes sufficiently rapidly as $|x| \rightarrow \infty$. More precisely, they modify $(1.2 \mathrm{~b})$ so that the initial data $u^{\varepsilon}(x, 0)$ is a reflectionless approximation to $v(x)$ that converges strongly to $v$ in $L^{2}$ as $\varepsilon \rightarrow 0$. The limit is characterized through a maximization problem parameterized by $(x, t)$ whose unique solution is a Lebesgue integrable function $\eta \mapsto \psi^{*}(\eta, x, t)$ over an interval $\eta \in\left[0, \eta_{\text {max }}\right]$. Weak limits of functionals of $u^{\varepsilon}$ and its derivatives can be expressed in terms of derivatives of moments of the measure $\psi^{*}(\eta, x, t) d \eta$. For example,

$$
\bar{u}(x, \mathbf{t}) \equiv \lim _{\varepsilon \rightarrow 0} u^{\varepsilon}(x, \mathbf{t})=\frac{4}{\pi} \int_{0}^{\eta_{\max }} \eta \partial_{x} \psi^{*}(\eta, x, \mathbf{t}) d \eta .
$$

Subsequently, Venakides found similar formulas for other classes of smooth initial data, including nonnegative data that vanishes sufficiently rapidly as $|x| \rightarrow \infty$ [25] and periodic data [27].

This paper begins with an appropriately normalized solution $f^{\varepsilon}(k, x, t)$ of $(1.3)$, the Jost function. We establish a direct relation between the maximizer $\psi^{*}(\cdot, x, t)$ and the zero-dispersion limit of the logarithm of the Jost functions. More specifically, we find that over all complex $k$ outside the imaginary interval $\left[-i \eta_{\max }, i \eta_{\max }\right]$ one has

$$
\lim _{\varepsilon \rightarrow 0} \varepsilon \log f^{\varepsilon}(k, x, \mathbf{t})=-i k x+p(i k, \mathbf{t})-\frac{1}{\pi} \int_{0}^{\eta_{\max }} \log \left(\frac{i k-\eta}{i k+\eta}\right)\left(\phi(\eta)-\psi^{*}(\eta, x, \mathbf{t})\right) d \eta,
$$

uniformly over compact subsets of $(k, x, t)$, where $\phi(\eta)$ is the Weyl asymptotic density of the eigenvalues for the Schrödinger operator $(1.3)$ and $p(\cdot, t)$ is the odd polynomial given by

$$
p(\eta, \mathbf{t})=\sum_{m=0}^{\infty} t_{m} 4^{m} \eta^{2 m+1} .
$$

Expanding both sides of (1.5) about $k=\infty$ then allows us to generate all the weak limit formulas in terms of moments of $\psi^{*}$. In the course of proving (1.5), we will be led to calculate the limit of certain spectral measures $d \sigma^{\varepsilon}(\eta ; x, t)$ associated with the Dirichlet problem for (1.3). More specifically, we shall show that

$$
\lim _{\varepsilon \rightarrow 0} d \sigma^{\varepsilon}(\eta ; x, \mathbf{t})=\psi^{*}(\eta, x, \mathbf{t}) d \eta,
$$

uniformly over compact subsets of $(x, t)$, thereby giving $\psi^{*}$ a direct spectral interpretation.

The paper is laid out as follows. Section 2 reviews the relevant facts about the $\mathrm{KdV}$ hierarchy and, by doing so, introduces our notational conventions. Section 3 poses the zero-dispersion limit problem and describes some strengthening of the main results of the Lax-Levermore theory. Section 4 introduces the Jost and Weyl functions and establishes the limit (1.5) for those imaginary $k$ for which $|k|>\eta_{\max }$. Section 5 develops the necessary background on Dirichlet spectrum, extends (1.5) 
to all complex $k$ outside of the imaginary interval [ $\left.-i \eta_{\max }, i \eta_{\max }\right]$, and derives (1.7). Section 6 discusses the limit as $\varepsilon \rightarrow 0$ of the Weyl functions

$$
m^{\varepsilon}(k, x, \mathbf{t}) \equiv \varepsilon \partial_{x} \log f^{\varepsilon}(k, x, \mathbf{t}),
$$

and derives information about the limiting form of the $\mathrm{KdV}$ conserved densities. Section 7 concludes with comments and discussion.

\section{The KdV Hierarchy}

The initial-value problem for the Korteweg-deVries (KdV) equation is

$$
\begin{gathered}
\partial_{t} u-6 u \partial_{x} u+\varepsilon^{2} \partial_{x x x} u=0, \\
u(x, 0)=v(x) .
\end{gathered}
$$

In this section $\varepsilon>0$ will be considered to be a fixed constant, and hence, no implicit $\varepsilon$ dependence will be indicated. This problem was first solved by Gardner, Greene, Kruskal and Miura [11] for initial data $v(x)$ that decays sufficiently rapidly as $|x| \rightarrow \infty$. Their critical observation was that the $\mathrm{KdV}$ equation (2.1) is the solvability condition for the linear system

$$
\begin{aligned}
\mathscr{L}(t) f & \equiv-\varepsilon^{2} \partial_{x x} f+u f=\lambda f, \\
\partial_{t} f=\mathscr{B}(t) f & \equiv-4 \varepsilon^{2} \partial_{x x x} f+3\left(u \partial_{x}+\partial_{x} u\right) f,
\end{aligned}
$$

where $\lambda$ is an eigenvalue of the Schrödinger operator $\mathscr{L}(t)$. Then given the potential $u(x, 0)$ of $\mathscr{L}(0)$, the asymptotics of the eigenfunctions $f$ as $|x| \rightarrow \infty$, referred to as the scattering data, can be calculated in principle. The evolution of the scattering data is determined explicitly and the potential $u(x, t)$ of $\mathscr{L}(t)$ is then obtained through the knowledge of the large $|x|$ asymptotics of $f$ using inverse scattering theory [7].

More specifically, for every $u$ in the scattering class the $L^{2}$-spectrum of the Schrödinger operator (2.2a) consists of the nonnegative semi-axis $\lambda \geqq 0$ along with a finite set (possibly empty) of negative simple eigenvalues $\lambda_{1}, \ldots, \lambda_{N}$. The asymptotic behavior of an eigenfunction $f=f(k, x)$ corresponding to a $\lambda=k^{2}>0$ in the continuous spectrum is given by

$$
f(k, x) \sim \begin{cases}\exp \left(\frac{-i k x}{\varepsilon}\right), & \text { for } x \rightarrow-\infty, \\ \frac{1}{T(k)} \exp \left(\frac{-i k x}{\varepsilon}\right)+\frac{R(k)}{T(k)} \exp \left(\frac{i k x}{\varepsilon}\right), & \text { for } x \rightarrow+\infty,\end{cases}
$$

where $T(k)$ and $R(k)$ are the so-called transmission and reflection coefficients. The asymptotic behavior of a real unit normalized eigenfunction $f=f_{j}(x)$ corresponding to a discrete eigenvalue $\lambda_{j}=-\eta_{j}^{2}<0$ is given by

$$
f_{j}(x) \sim \exp \left(\frac{-\eta_{j} x+\chi_{j}}{\varepsilon}\right), \quad \text { for } x \rightarrow+\infty .
$$

The inverse theory prescribes that the fundamental scattering data consist of the reflection coefficient $R(k)$, the eigenvalues $\lambda_{j}$, and the norming exponents $\chi_{j}$. The 
transmission coefficient $T(k)$, as well as all other asymptotic information, can be computed from this fundamental set.

As $u$ evolves according to the $\mathrm{KdV}$ equation (2.1a) then for every $\lambda=k^{2}$ in the continuous spectrum there is a solution $f(k, x, t)$ of the linear system (2.2) that evolves as

$$
f(k, x, t) \sim \begin{cases}\exp \left(\frac{-i k x-4 i k^{3} t}{\varepsilon}\right), & \text { for } x \rightarrow-\infty, \\ \frac{1}{T(k)} \exp \left(\frac{-i k x-4 i k^{3} t}{\varepsilon}\right)+\frac{R(k)}{T(k)} \exp \left(\frac{i k x+4 i k^{3} t}{\varepsilon}\right), & \text { for } x \rightarrow+\infty,\end{cases}
$$

while the eigenvalues $\lambda_{j}=-\eta_{j}^{2}$ remain fixed in time and there is a solution $f_{j}(x, t)$ of (2.2) that evolves as

$$
f_{j}(x, t) \sim \exp \left(\frac{-\eta_{j} x+4 \eta_{j}^{3} t+\chi_{j}}{\varepsilon}\right), \text { for } x \rightarrow+\infty,
$$

where the $T(k), R(k)$, and $\chi_{j}$ are the initial scattering data. Comparing (2.5) and (2.6) with (2.3) and (2.4), the time evolution of the scattering data for $u(x, t)$ can be read off as

$$
\chi_{j}(t)=\chi_{j}+4 \eta_{j}^{3} t, \quad R(k, t)=R(k) \exp \left(i \frac{8 k^{3} t}{\varepsilon}\right) .
$$

Hence, given $R(k), \eta_{j}$, and $\chi_{j}$ computed from the initial data $u(x, 0)$, the solution $u(x, t)$ of the $\mathrm{KdV}$ equation (2.1) is then determined by inverse scattering from the $R(k, t), \eta_{j}$, and $\chi_{j}(t)$ given by (2.7).

Given the solution $f(k, x, t)$ of system (2.2) that satisfies the asymptotics (2.5), we introduce the Weyl function, $m(k, x, t)$, by

$$
m \equiv \varepsilon \partial_{x} \log f \text {. }
$$

A direct calculation starting from (2.2a) shows that $m$ satisfies the Ricatti equation

$$
-\varepsilon \partial_{x} m=k^{2}-u+m^{2} \text {. }
$$

Proceeding from (2.2b) while using (2.9) to eliminate all explicit occurrences of $u$ yields

$$
-\varepsilon \partial_{t} \log f=-6 k^{2} m-2 m^{3}+\varepsilon^{2} \partial_{x x} m .
$$

Cross-differentiating (2.8) and (2.10) shows that $m$ satisfies the modified KdV equation [12]

$$
\partial_{t} m+\partial_{x}\left(-6 k^{2} m-2 m^{3}+\varepsilon^{2} \partial_{x x} m\right)=0 .
$$

This single local conservation law depends on the parameter $k$. The analytic dependence of the Jost function on $k$ [3] gives the expansions

$$
\begin{aligned}
m & =\varepsilon \partial_{x} \log f=-i k+\sum_{\ell=0}^{\infty} \frac{\rho_{\ell}}{(2 i k)^{\ell+1}}, \\
-6 k^{2} m-2 m^{3}+\varepsilon^{2} \partial_{x x} m & =-\varepsilon \partial_{t} \log f=i 4 k^{3}+\sum_{\ell=0}^{\infty} \frac{v_{\ell}}{(2 i k)^{\ell+1}} .
\end{aligned}
$$


Placing these expansions into the modified KdV equation (2.11) then yields

$$
\partial_{t} \rho_{\ell}+\partial_{x} v_{\ell}=0, \quad \text { for } \ell=0,1, \ldots,
$$

thereby indicating that the KdV equation (2.1a) may possess an infinite family of nontrivial local conservation laws with densities $\rho_{\ell}$ and fluxes $v_{\ell}$.

The densities $\rho_{\ell}$ are computed in terms of $u$ by formally substituting the expansion (2.12a) into the Ricatti equation (2.9). The first five densities are found to be

$$
\begin{aligned}
& \rho_{0}=-u, \\
& \rho_{1}=-\varepsilon \partial_{x} u, \\
& \rho_{2}=u^{2}-\varepsilon^{2} \partial_{x x} u, \\
& \rho_{3}=4 \varepsilon u \partial_{x} u-\varepsilon^{3} \partial_{x x x} u, \\
& \rho_{4}=-2 u^{3}+6 \varepsilon^{2} u \partial_{x x} u+5 \varepsilon^{2}\left(\partial_{x} u\right)^{2}-\varepsilon^{4} \partial_{x x x x} u .
\end{aligned}
$$

In general, the coefficients $\rho_{\ell}$ satisfy the recursion relation

$$
\rho_{\ell+1}=\sum_{j=1}^{\ell} \rho_{\ell-j} \rho_{j-1}+\varepsilon \partial_{x} \rho_{\ell}, \quad \text { for } \ell=1,2, \ldots
$$

Notice that $\rho_{1}$ and $\rho_{3}$ are perfect derivatives, a property shared by all the densities with odd indices. This can be seen by decomposing $m$ into $m_{e}$ and $m_{o}$, its even and odd components as a function of $k$, and observing that the odd component of the Ricatti equation (2.9) gives the relation

$$
m_{e}=-\frac{1}{2} \varepsilon \partial_{x} \log m_{o}
$$

Hence, only the densities $\rho_{2 n}$, for $n=0,1, \ldots$, can give nontrivial local conservation laws, which in fact they do [17]. Of course, any of these densities could be modified by a multiplicative constant or an additive perfect derivative without changing any of the essential mathematics, but the normalization adopted here in terms of the expansions (2.12) is the most natural for our purposes.

Associated with each of the nontrivial locally conserved densities $\rho_{2 n}$ is a conserved functional $H_{n}$ that can be expressed as

$$
H_{n}=\int_{-\infty}^{\infty} \frac{1}{2} \rho_{2 n} d x, \text { for } n=0,1, \ldots
$$

The first three conserved functionals $H_{n}$ so obtained from (2.14) are simply

$$
H_{0}=-\int_{-\infty}^{\infty} \frac{1}{2} u d x, \quad H_{1}=\int_{-\infty}^{\infty} \frac{1}{2} u^{2} d x, \quad H_{2}=-\int_{-\infty}^{\infty} u^{3}+\frac{1}{2} \varepsilon^{2}\left(\partial_{x} u\right)^{2} d x .
$$

The KdV equation (2.1a) can be recast in the Hamiltonian form

$$
\partial_{t} u+\partial_{x} \frac{\delta H_{2}}{\delta u}=0
$$


where the Hamiltonian is $\mathrm{H}_{2}$ given in (2.18). The infinite family of conserved functionals $H_{n}$ are independent and satisfy the Poisson commutation relation

$$
0=\left\{H_{n}, H_{m}\right\} \equiv \int_{-\infty}^{\infty} \frac{\delta H_{n}}{\delta u} \partial_{x} \frac{\delta H_{m}}{\delta u} d x, \quad \text { for } n, m=0,1, \ldots,
$$

and are therefore in involution [10,32]. Each $H_{m}$ except $H_{0}$ is a Hamiltonian which generates a member of the so-called $\mathrm{KdV}$ hierarchy of commuting flows [17]. Let $t_{m}$ denote the time variable associated with the $m^{\text {th }} \mathrm{KdV}$ flow as generated by $H_{m+1}$ through the equation

$$
\partial_{t_{m}} u+\partial_{x} \frac{\delta H_{m+1}}{\delta u}=0, \quad \text { for } m=0,1, \ldots
$$

Recalling $H_{1}$ and $H_{2}$ from (2.18), for $m=0$ Eq. (2.21) is the flow of positive $x$-translation by $t_{0}$ while for $m=1$ it is the KdV flow (2.19) with $t_{1}$ being identified with $t$. This observation motivated the introduction of the $\frac{1}{2}$ into (2.17). By the Poisson commutation (2.20), every $H_{n}$ is conserved by each of these flows.

Because these flows commute, they may be solved simultaneously for $u=u(x, t)$ where, for the moment, the time vector $\mathbf{t}=\left(t_{0}, t_{1}, \ldots\right)$ is understood to have all but finitely many $t_{m}$ zero. Associated with each such $t$ is the odd polynomial $p(\cdot, t)$ defined by

$$
p(\eta, \mathbf{t})=\sum_{m=0}^{\infty} t_{m} 4^{m} \eta^{2 m+1} .
$$

The simultaneous evolution of the scattering data is then given by

$$
\chi_{j}(\mathbf{t})=\chi_{j}+p\left(\eta_{j}, \mathbf{t}\right), \quad R(k, \mathbf{t})=R(k) \exp \left(\frac{-2 p(i k, \mathbf{t})}{\varepsilon}\right),
$$

and $u(x, \mathbf{t})$ is determined by inverse scattering. This solution may be extended to all those time vectors $\mathbf{t}$ in the class

$$
\mathscr{E} \equiv\left\{\mathbf{t}: \lim _{m \rightarrow \infty}\left|t_{m}\right|^{\frac{1}{m}}=0\right\},
$$

which corresponds to extending the class of mappings $\eta \mapsto p(\eta, t)$ to those that are entire, odd, and have real symmetry.

Under the $m^{\text {th }}$ flow the density $\rho_{2 n}$ satisfies the local conservation law

$$
\partial_{t_{m}} \rho_{2 n}+\partial_{x} v_{2 m, 2 n}=0, \quad \text { for } m, n=0,1, \ldots .
$$

The flux $v_{2 m, 2 n}$ for the density $\rho_{2 n}$ under the $m^{\text {th }}$ flow is determined by the expansion

$$
-\varepsilon \partial_{t_{m}} \log f=-4^{m}(i k)^{2 m+1}+\sum_{\ell=0}^{\infty} \frac{v_{2 m, \ell}}{(2 i k)^{\ell+1}} .
$$

Note that for $m=0$, corresponding to the $x$-translational flow, one has $v_{0, \ell}=\rho_{\ell}$, while for $m=1$, corresponding to the $\mathrm{KdV}$ flow, one has $v_{2, \ell}=v_{\ell}$ as determined by (2.12b). By setting $n=0$ in (2.25) one recovers the $m^{\text {th }}$ flow (2.21) in the KdV hierarchy.

There is a deep relation [8] between the coefficients in the expansion (2.12a) and the coefficients in the expansions in (2.26). After some rescaling this relation 
is equivalent to

$$
(2 n+1) \rho_{2 n}-v_{2 n, 0}=\text { a perfect } x \text {-derivative . }
$$

As stated earlier, because an additive perfect derivative does not alter the essential mathematics of the conservation laws, $(2 n+1) \rho_{2 n}$ and $v_{2 n, 0}$ are densities for the same conserved quantity.

\section{The Zero-Dispersion Limit}

The problem of the zero-dispersion limit for the $\mathrm{KdV}$ considers the solution $u^{\varepsilon}(x, t)$ of the whole $\mathrm{KdV}$ hierarchy (2.9) as a function of $\varepsilon$ for some initial data $v(x)$ that is independent of $\varepsilon$, and tries to determine the limiting behavior of the conserved densities $\rho_{n}^{\varepsilon}$ and fluxes $v_{m, n}^{\varepsilon}$ as $\varepsilon$ tends to zero. Lax and Levermore $[18,19]$ analyzed the limiting behavior of the scattering and inverse scattering transform using a WKB analysis of (2.2) and a kind of steepest descent argument to obtain a characterization of the (weak) limits in terms of the solution of a variational problem. Below we recall those aspects of this theory that are relevant to this investigation.

For simplicity, we present only the case when the initial data $v(x)$ is a single nonpositive well with a minimum value of $-\eta_{\max }^{2}$ as depicted in Fig. 3.1. Possible extensions of our results to other classes of initial data will be discussed in the conclusions.

For small $\varepsilon$ approximate scattering data of $v(x)$ may be computed using the WKB method. The WKB turning point analysis yields eigenvalues $\lambda_{j}^{\varepsilon}=-\eta_{j}^{\varepsilon 2}$ that are distributed within the open interval $\left(-\eta_{\max }^{2}, 0\right)$ so as to be consistent with the socalled Weyl asymptotic density $\varphi(\eta)$ with respect to the spectral variable $\eta$, which is given by

$$
\varphi(\eta)=\int_{x_{-}(\eta)}^{x_{+}(\eta)} \frac{\eta}{\sqrt{-v(x)-\eta^{2}}} d x
$$

where $x_{-}(\eta)<x_{+}(\eta)$ are determined by $v\left(x_{ \pm}\right)=-\eta^{2}$ as shown in Fig. 3.1. Specifically, $\eta=\eta_{j}^{\varepsilon}$ is the unique positive solution of

$$
\frac{1}{\pi \varepsilon} \Phi(\eta)=j-\frac{1}{2}, \quad \text { for } j=1, \ldots, N^{\varepsilon},
$$

where $\Phi(\eta)$ is defined by

$$
\Phi(\eta) \equiv \int_{\eta}^{\eta_{\max }} \varphi(\xi) d \xi=\int_{x_{-}(\eta)}^{x_{+}(\eta)} \sqrt{-v(x)-\eta^{2}} d x
$$

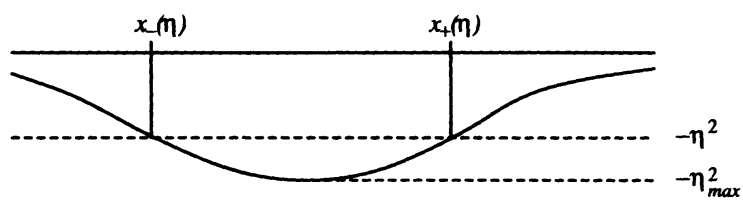

Fig. 3.1 A typical initial data $v=v(x)$ considered here. The defining relations for the functions $x=x_{ \pm}(\eta)$ are also indicated 
and $N^{\varepsilon}$ is defined by

$$
N^{\varepsilon} \equiv \operatorname{Int}\left[\frac{1}{\pi \varepsilon} \Phi(0)\right]=\operatorname{Int}\left[\frac{1}{\pi \varepsilon} \int_{-\infty}^{\infty} \sqrt{-v(x)} d x\right] .
$$

Here Int $[\cdot]$ rounds its argument to the closest integer value, with half-integers rounded down. So determined, the WKB eigenvalues are strictly ordered as

$$
-\eta_{\max }^{2}<\lambda_{1}^{\varepsilon}<\cdots<\lambda_{j}^{\varepsilon}<\cdots<\lambda_{N^{\varepsilon}}^{\varepsilon}<0 .
$$

The corresponding norming exponents obtained from the WKB analysis are

$$
\chi_{j}^{\varepsilon}=\chi\left(\eta_{j}^{\varepsilon}\right), \quad \text { for } j=1, \ldots, N^{\varepsilon},
$$

where the so-called asymptotic norming exponent $\chi(\eta)$ is defined by

$$
\chi(\eta)=\eta x_{+}(\eta)+\int_{x_{+}(\eta)}^{\infty}\left(\eta-\sqrt{\eta^{2}+v(x)}\right) d x .
$$

Finally, the reflection coefficient is found to be zero to all orders.

Motivated by this calculation, we choose to replace the exact initial data $v(x)$ by the reflectionless potential $v^{\varepsilon}(x)$ corresponding to the above WKB scattering data. While this device is not the best one might hope for, it is partially justified a posteriori by the result that $v^{\varepsilon}$ converges strongly to $v$. The solution $u^{\varepsilon}(x, t)$ of the whole $\mathrm{KdV}$ hierarchy corresponding to this reflectionless initial data can be constructed from the approximate scattering data by the Kay-Moses formula [16]

$$
u^{\varepsilon}(x, \mathbf{t})=-2 \varepsilon^{2} \partial_{x x} \log \tau^{\varepsilon}(x, \mathbf{t}),
$$

where the so-called tau-function $\tau^{\varepsilon}(x, \mathbf{t})$ is the $N^{\varepsilon} \times N^{\varepsilon}$ determinant

$$
\tau^{\varepsilon}(x, \mathbf{t})=\operatorname{det}\left(I+G^{\varepsilon}(x, \mathbf{t})\right) .
$$

Here the matrix $G^{\varepsilon}$ has the form

$$
G^{\varepsilon}(x, \mathbf{t})=\left(\frac{1}{\eta_{j}^{\varepsilon}+\eta_{l}^{\varepsilon}} \exp \left(\frac{a\left(\eta_{j}^{\varepsilon}, x, \mathbf{t}\right)+a\left(\eta_{l}^{\varepsilon}, x, \mathbf{t}\right)}{\varepsilon}\right)\right),
$$

where

$$
a(\eta, x, \mathbf{t}) \equiv-\eta x+p(\eta, \mathbf{t})+\chi(\eta) .
$$

Associated conserved densities and fluxes are given by $[8,22,31]$

$$
\begin{aligned}
\frac{2 n+1}{2} \rho_{2 n}^{\varepsilon}(x, \mathbf{t}) & =-\varepsilon^{2} \partial_{x t_{n}} \log \tau^{\varepsilon}(x, \mathbf{t}), \\
\frac{2 m+1}{2} v_{2 n, 2 m}^{\varepsilon}(x, \mathbf{t}) & =\varepsilon^{2} \partial_{t_{n} t_{m}} \log \tau^{\varepsilon}(x, \mathbf{t}) .
\end{aligned}
$$

It is important to understand that these are not the same densities and fluxes as were defined in (2.12a) and (2.26) respectively. They do, however, give the same integrated conservation laws as a consequence of (2.27). 
The Kay-Moses formula (3.8) is the special case of (3.12a) obtained by setting $n=0$ while using (2.14) and the fact that $\partial_{t_{0}} \tau^{\varepsilon}=-\partial_{x} \tau^{\varepsilon}$. Moreover, a comparison of (3.12a) with (3.12b) when $m=0$ yields

$$
(2 n+1) \rho_{2 n}^{\varepsilon}=-2 \varepsilon^{2} \partial_{x t_{n}} \log \tau^{\varepsilon}(x, \mathbf{t})=2 \varepsilon^{2} \partial_{t_{0} t_{n}} \log \tau^{\varepsilon}(x, \mathbf{t})=v_{2 n, 0}^{\varepsilon} .
$$

This is equivalent to (2.27) modulo the freedom to add a perfect $x$-dervative to the conserved densities which does not alter the essential content of the conservation laws.

The class of $\mathbf{t}$ for which the above constructions are valid may be extended from the class $\mathscr{E}$ of $(2.24)$ to the real-valued weighted $\ell^{1}$ space

$$
\mathscr{T} \equiv\left\{\mathrm{t}: \sum_{m=0}^{\infty}\left|t_{m}\right| 4^{m} \eta_{\max }^{2 m+1}<\infty\right\}
$$

The space $\mathscr{T}$ shall be considered as a representation of the dual space of $c_{0}$, the space of sequences that converge to zero, and, as such, to be endowed with its weak-* topology. With this topology for $\mathscr{T}$ the matrix $G^{\varepsilon}$ and the function $\tau^{\varepsilon}$ are clearly continuous over $\mathbb{R} \times \mathscr{T}$.

At this point we make the important observation that for each $\varepsilon>0$ the function

$$
(x, t) \mapsto \log \tau^{\varepsilon}(x, t) \quad \text { is smooth, positive and convex . }
$$

These properties are all consequences of the fact that the matrix $G^{\varepsilon}(x, t)$ of $(3.10)$ is Hermitian positive [19], which by (3.9) immediately gives that $\tau^{\varepsilon}(x, t)>1$. The regularity and positivity are then obvious. The convexity was first noticed in the context of the semiclassical limit of the nonlinear Schrödinger equation [14,15], but the proof applies to the $\mathrm{KdV}$ case without change. Indeed, this convexity will hold for any tau-function, $\tau^{\varepsilon}(x, t)$, that is a linear combination of real exponentials with positive coefficients.

The Lax-Levermore theory $[18,19]$ establishes the existence of the limit

$$
\lim _{\varepsilon \rightarrow 0} \varepsilon^{2} \log \tau^{\varepsilon}(x, \mathbf{t})=q(x, \mathbf{t}),
$$

where the limit is uniform over compact subsets of $(x, t)$ in $\mathbb{R} \times \mathscr{T}$. Moreover, the limit $q(x, t)$ is characterized by the maximization problem

$$
q(x, \mathbf{t})=\frac{2}{\pi} \max \{Q(\psi ; x, \mathbf{t}): \psi \in \mathscr{A}\},
$$

where the admissible set $\mathscr{A}$ is defined by

$$
\mathscr{A} \equiv\left\{\psi \in L^{1}\left(\left[0, \eta_{\max }\right]\right): 0 \leqq \psi \leqq \varphi\right\},
$$

and the quadratic functional $Q(\psi ; x, t)$ is defined by

$$
\begin{aligned}
Q(\psi ; x, \mathbf{t})= & \frac{2}{\pi} \int_{0}^{\eta_{\max }} a(\eta, x, \mathbf{t}) \psi(\eta) d \eta \\
& +\frac{1}{\pi^{2}} \int_{0}^{\eta_{\max }} \int_{0}^{\eta_{\max }} \log \left|\frac{\eta-\xi}{\eta+\xi}\right| \psi(\eta) \psi(\xi) d \eta d \xi,
\end{aligned}
$$


with $a(\eta, x, t)$ given by (3.11). The initial data $v(x)$ enters this problem through the Weyl asymptotic density $\varphi(\eta)$ defined by (3.1), which determines the admissible set $\mathscr{A}$ in (3.17), and through the asymptotic norming exponent $\chi(\eta)$ defined by (3.7), which arises in the $a(\eta, x, t)$ appearing in (3.18). For every $(x, t) \in \mathbb{R} \times \mathscr{T}$ the functional $Q(\psi ; x, t)$ is bounded above and strictly concave over $\psi \in \mathscr{A}$. Hence, its maximum value $q(x, \mathbf{t})$ is attained at a unique $\psi=\psi^{*}(\cdot, x, \mathbf{t})$ in the admissible set $\mathscr{A}$ that depends continuously on $(x, t)$ when identifying $\mathscr{A}$ as a set of densities endowed with the weak topology of measures. Moreover, this value is nonnegative because (3.17) shows 0 to be in the admissible set $\mathscr{A}$.

The uniqueness and continuity of $\psi^{*}$ imply [19] that the maximum $q(x, t)$ is a continuously differentiable function of $(x, t)$ with

$$
\begin{aligned}
& \partial_{x} q(x, \mathbf{t})=-\frac{2}{\pi} \int_{0}^{\eta_{\max }} \eta \psi^{*}(\eta, x, \mathbf{t}) d \eta \\
& \partial_{\mathrm{t}} q(x, \mathbf{t})=\frac{2}{\pi} \int_{0}^{\eta_{\max }} p_{\mathrm{t}}(\eta) \psi^{*}(\eta, x, \mathbf{t}) d \eta
\end{aligned}
$$

where $p_{\mathbf{t}}(\eta) \equiv \partial_{\mathbf{t}} p(\eta, \mathbf{t})$ is independent of $\mathbf{t}$. It is an elementary, but nontrivial, fact that the regularity and convexity of both the approximating functions $\varepsilon^{2} \log \tau^{\varepsilon}(3.14)$ and the limiting function $q$ in (3.15) imply [15] that

$$
\begin{aligned}
& \lim _{\varepsilon \rightarrow 0} \varepsilon^{2} \partial_{x} \log \tau^{\varepsilon}(x, \mathbf{t})=\partial_{x} q(x, \mathbf{t}), \\
& \lim _{\varepsilon \rightarrow 0} \varepsilon^{2} \partial_{t} \log \tau^{\varepsilon}(x, \mathbf{t})=\partial_{\mathbf{t}} q(x, \mathbf{t}),
\end{aligned}
$$

where again the limits are uniform over compact subsets of $(x, t)$ in $\mathbb{R} \times \mathscr{T}$. Hence, the convergence of $\varepsilon^{2} \log \tau^{\varepsilon}$ to $q$ in (3.15) is in $C^{1}(\mathbb{R} \times \mathscr{T})$. In deriving the main result of the next section, we will make full use of this $C^{1}$ convergence.

Because the map $(x, t) \mapsto q(x, t)$ is realized as a supremum of linear functions of $(x, t)$, it is convex. The regularity and convexity of the function $q$ also imply that its Hessian matrix of distributional second derivatives is a measurable function that is given by a Hessian matrix of classical derivatives almost everywhere. Its values in terms of $\psi^{*}$ are given by

$$
\begin{aligned}
& \left(\begin{array}{ll}
\partial_{x x} q(x, \mathbf{t}) & \partial_{x t} q(x, \mathbf{t}) \\
\partial_{\mathbf{t} x} q(x, \mathbf{t}) & \partial_{\mathrm{tt}} q(x, \mathbf{t})
\end{array}\right) \\
& \quad=\left(\begin{array}{ll}
-\frac{2}{\pi} \int_{0}^{\eta_{\max }} \eta \partial_{x} \psi^{*}(\eta, x, \mathbf{t}) d \eta & -\frac{2}{\pi} \int_{0}^{\eta_{\max }} \eta \partial_{\mathbf{t}} \psi^{*}(\eta, x, \mathbf{t}) d \eta \\
\frac{2}{\pi} \int_{0}^{\eta_{\max }} p_{\mathbf{t}}(\eta) \partial_{x} \psi^{*}(\eta, x, \mathbf{t}) d \eta & \frac{2}{\pi} \int_{0}^{\eta_{\max }} p_{\mathbf{t}}(\eta) \partial_{\mathbf{t}} \psi^{*}(\eta, x, \mathbf{t}) d \eta
\end{array}\right),
\end{aligned}
$$

where the derivatives on $\psi^{*}$ are understood in the sense of distributions. 
Combining (3.20) and (3.21) with (3.12) leads to the weak limits

$$
\begin{aligned}
\frac{2 n+1}{2} \lim _{\varepsilon \rightarrow 0} \rho_{2 n}^{\varepsilon}(x, \mathbf{t})=-\partial_{x t_{n}} q(x, \mathbf{t}) & =-\frac{2}{\pi} \int_{0}^{\eta_{\max }} p_{t_{n}}(\eta) \partial_{x} \psi^{*}(\eta, x, \mathbf{t}) d \eta \\
& =\frac{2}{\pi} \int_{0}^{\eta_{\max }} \eta \partial_{t_{n}} \psi^{*}(\eta, x, \mathbf{t}) d \eta, \\
\frac{2 n+1}{2} \lim _{\varepsilon \rightarrow 0} v_{2 m, 2 n}^{\varepsilon}(x, \mathbf{t})=\partial_{t_{m} t_{n}} q(x, \mathbf{t}) & =\frac{2}{\pi} \int_{0}^{\eta_{\max }} p_{t_{n}}(\eta) \partial_{t_{m}} \psi^{*}(\eta, x, \mathbf{t}) d \eta \\
& =\frac{2}{\pi} \int_{0}^{\eta_{\max }} p_{t_{m}}(\eta) \partial_{t_{n}} \psi^{*}(\eta, x, \mathbf{t}) d \eta,
\end{aligned}
$$

for the conserved densities and fluxes associated with the solution $u^{\varepsilon}(x, t)$. The above limits for $\rho_{2 n}^{\varepsilon}$ are weak with respect to the $x$ variable, while those for $v_{2 m, 2 n}^{\varepsilon}$ are weak with respect to the $t_{m}$ variable. In particular, (3.22) gives

$$
\lim _{\varepsilon \rightarrow 0} u^{\varepsilon}(x, \mathbf{t})=-2 \partial_{x x} q(x, \mathbf{t})=\frac{4}{\pi} \int_{0}^{\eta_{\max }} \eta \partial_{x} \psi^{*}(\eta, x, \mathbf{t}) d \eta,
$$

in the sense of $x$-distributions, which resolves the question raised in (1.1). We remark that the general limit formulas for all conserved densities and fluxes (3.22) are not found in $[18,19]$, but first appeared in the context of the semiclassical limit of the defocusing nonlinear Schrödinger equation [13-15]. However, these generalizations are straightforward given (3.12) once the strong limit of $\varepsilon^{2} \log \tau^{\varepsilon}$ is established as was done in [19].

The significance of this result is that it provides a global characterization of the zero-dispersion limit for all the conserved densities and fluxes of the $\mathrm{KdV}$ hierarchy as a function of the initial data through the maximization problem (3.16). Of course, the story of the zero-dispersion limit goes far beyond this characterization. The variational problem associated with (3.16) was transformed into a Riemann-Hilbert problem and systems of hyperbolic equations were found that locally describe the evolution of the zero-dispersion limit $[18,19,21]$. These so-called Whitham modulation equations were found independently by Flaschka, Forest and McLaughlin [9] by the averaging of families of quasi-periodic KdV solutions, thereby generalizing the early work of Whitham $[29,30]$. While this modulation approach provides a far better picture of what the $\mathrm{KdV}$ solution might look like for $\varepsilon$ small, it is intrinsically local in nature and cannot connect regions in $(x, t)$-space that are governed by the averaging of different families of $\mathrm{KdV}$ solutions. In particular, it does not relate the dynamics back to the initial data. On the other hand, the global nature of the Lax-Levermore solution does provide a prescription of how the modulation equations arising from averaging different families partition $(x, t)$ space [19]. Venakides [26-28] helped bridge the gap between these two approaches by developing a theory of the microstructure of solutions based on quantizing the Lax-Levermore variational problem. All of these results and more are surveyed in $[4,20]$.

\section{The Behavior of the Jost Function}

Given any solution of the $\mathrm{KdV}$ hierarchy $u^{\varepsilon}(x, \mathbf{t})$, there is a unique eigenfunction $f^{\varepsilon}(k, x, \mathbf{t})$ of the associated Schrödinger operator $\mathscr{L}^{\varepsilon}(\mathbf{t})$ that is analytic in the 
upper-half complex $k$-plane and satisfies

$$
\mathscr{L}^{\varepsilon}(\mathbf{t}) f^{\varepsilon} \equiv-\varepsilon^{2} \partial_{x x} f^{\varepsilon}+u^{\varepsilon}(x, \mathbf{t}) f^{\varepsilon}=k^{2} f^{\varepsilon},
$$

and has the large $k$ expansion of

$$
f^{\varepsilon}(k, x, \mathbf{t})=\exp \left(\frac{-i k x+p(i k, \mathbf{t})}{\varepsilon}\right)\left(1+\frac{b_{1}^{\varepsilon}(x, \mathbf{t})}{i k}+\cdots+\frac{b_{n}^{\varepsilon}(x, \mathbf{t})}{(i k)^{n}}+\cdots\right) .
$$

This function is known as the Jost function of $\mathscr{L}^{\varepsilon}(\mathbf{t})$ [3].

When $u^{\varepsilon}$ is the reflectionless potential given by (3.8), the Jost function can be expressed in terms of the scattering data [3] as

$$
f^{\varepsilon}(k, x, \mathbf{t})=\exp \left(\frac{-i k x+p(i k, \mathbf{t})}{\varepsilon}\right) \frac{\tau^{\varepsilon}(i k, x, \mathbf{t})}{\tau^{\varepsilon}(x, \mathbf{t})} \prod_{j=1}^{N^{\varepsilon}} \frac{i k+\eta_{j}^{\varepsilon}}{i k-\eta_{j}^{\varepsilon}},
$$

where the tau-function $\tau^{\varepsilon}(x, t)$ is given by (3.9) and the $k$-dependent tau-function $\tau^{\varepsilon}(i k, x, \mathbf{t})$ is defined by the $N^{\varepsilon} \times N^{\varepsilon}$ determinant

$$
\tau^{\varepsilon}(i k, x, \mathbf{t})=\operatorname{det}\left(I+D^{\varepsilon}(i k) G^{\varepsilon}(x, \mathbf{t}) D^{\varepsilon}(i k)\right)=\operatorname{det}\left(I+D^{\varepsilon}(i k)^{2} G^{\varepsilon}(x, \mathbf{t})\right),
$$

with $G^{\varepsilon}(x, t)$ as in (3.10) and the diagonal matrix $D^{\varepsilon}(i k)$ defined by

$$
D^{\varepsilon}(i k)=\operatorname{diag}\left(\sqrt{\frac{i k-\eta_{j}^{\varepsilon}}{i k+\eta_{j}^{\varepsilon}}}\right) .
$$

Here the square root is taken on the sheet where $\sqrt{1}=1$ and with its branch cut on the negative real axis so that $D^{\varepsilon}(i k)$ is analytic off the imaginary interval $\left[-i \eta_{\max }, i \eta_{\max }\right]$.

Clearly, (4.4) shows $\tau^{\varepsilon}(i k, x, t)$ to be a rational function of $k$ which has the asymptotic value of $\tau^{\varepsilon}(x, t)$ as $k \rightarrow \infty$ and possesses the symmetry

$$
\overline{\tau^{\varepsilon}(-i \bar{k}, x, \mathbf{t})}=\tau^{\varepsilon}(i k, x, \mathbf{t})
$$

thereby taking on real values along the imaginary axis everywhere it is defined. Its denominator has simple zeros which are fixed at $k=i \eta_{j}^{\varepsilon}$ for $j=1, \ldots, N^{\varepsilon}$ for every $(x, t)$. Hence, after removing any removable singularities, $\tau^{\varepsilon}(i k, x, t)$ can have at most $N^{\varepsilon}$ simple poles. Less obvious is the fact that the $N^{\varepsilon}$ zeros of its numerator, which depend on $(x, t)$, always lie on the imaginary interval [-i $\left.\eta_{\max }, i \eta_{\max }\right]$. This is because $f^{\varepsilon}(k, x, t)$ can only vanish for those $k$ that lie inside the interval $\left[-i \eta_{\max }, i \eta_{\max }\right]$. To see this last fact, first note that, so long as $k$ is not a pole, the asymptotic behavior of $f^{\varepsilon}(k, x, t)$ for large values of $x$ is given by

$$
f^{\varepsilon}(k, x, \mathbf{t}) \sim \begin{cases}\exp \left(\frac{-i k x+p(i k, \mathbf{t})}{\varepsilon}\right), & \text { for } x \rightarrow-\infty, \\ \exp \left(\frac{-i k x+p(i k, \mathbf{t})}{\varepsilon}\right) \prod_{j=1}^{N^{\varepsilon}} \frac{i k+\eta_{j}^{\varepsilon}}{i k-\eta_{j}^{\varepsilon}}, & \text { for } x \rightarrow+\infty .\end{cases}
$$


When $k$ is nonzero and real it follows from (4.7) that $f^{\varepsilon}$ and $\overline{f^{\varepsilon}}$ are a pair of linearly independent solutions of the Schrödinger equation (4.1) with Wronskian

$$
\mathscr{W}\left(f^{\varepsilon}, \overline{f^{\varepsilon}}\right) \equiv f^{\varepsilon}(k, x, \mathbf{t}) \partial_{x} \overline{f^{\varepsilon}}(k, x, \mathbf{t})-\overline{f^{\varepsilon}}(k, x, \mathbf{t}) \partial_{x} f^{\varepsilon}(k, x, \mathbf{t})=\frac{2 i k}{\varepsilon}
$$

In that case it is clear from $(4.8)$ that $f^{\varepsilon}(k, x, t)$ can never vanish. On the other hand, if $f^{\varepsilon}\left(k_{o}, x_{o}, t_{o}\right)=0$ for some $k_{o}$ that lies outside the interval [ $-i \eta_{\max }, i \eta_{\max }$ ] and inside either the lower or the upper open half-plane then $k_{o}^{2}$ will be an eigenvalue with eigenfunction $f^{\varepsilon}\left(k_{o}, x, t_{o}\right)$ for the Schrödinger operator with potential $u\left(x, \mathbf{t}_{o}\right)$ and a Dirichlet boundary condition at $x_{o}$ considered over $L^{2}\left(\left[x_{o}, \infty\right)\right)$ or $L^{2}\left(\left(-\infty, x_{o}\right]\right)$ respectively. These operators are selfadjoint, so $k_{o}^{2}$ must be real and, hence, $k_{o}$ must be imaginary. However, for every $k$ that is imaginary and lies outside the interval $\left[-i \eta_{\max }, i \eta_{\max }\right]$, the diagonal matrix $D^{\varepsilon}(i k)$ will have positive entries and, therefore, $G^{\varepsilon}(x, t)$ will be Hermitian positive. It then follows from (4.4) that $\tau^{\varepsilon}(i k, x, t)>1$. Hence, $f^{\varepsilon}(k, x, t)$ can only vanish for those $k$ that lie inside the interval $\left[-i \eta_{\max }, i \eta_{\max }\right]$.

The Jost function can be related directly to the conserved densities and fluxes of the $\mathrm{KdV}$ hierarchy through the large $k$ asymptotics of its logarithmic derivatives. Specifically,

$$
\begin{aligned}
\varepsilon \partial_{x} \log f^{\varepsilon}(k, x, \mathbf{t}) & =-i k+\sum_{\ell=0}^{\infty} \rho_{\ell}^{\varepsilon}(x, \mathbf{t})\left(\frac{1}{2 i k}\right)^{\ell+1} \\
-\varepsilon \partial_{t_{m}} \log f^{\varepsilon}(k, x, \mathbf{t}) & =-4^{m}(i k)^{2 m+1}+\sum_{\ell=0}^{\infty} v_{2 m, \ell}^{\varepsilon}(x, \mathbf{t})\left(\frac{1}{2 i k}\right)^{\ell+1} .
\end{aligned}
$$

Therefore, the limiting form for all these quantities will be encoded in the limiting form of $\varepsilon \log f^{\varepsilon}(k, x, \mathbf{t})$. By the argument in the previous paragraph, this function is analytic over all complex $k$ outside the imaginary interval [-i $\left.\eta_{\max }, i \eta_{\max }\right]$, that is, in $\Omega \equiv\left\{k \in \mathbb{C}: k \notin\left[-i \eta_{\max }, i \eta_{\max }\right]\right\}$. As will be shown below, the limit of this function can be evaluated in terms of the maximizer $\psi^{*}(\eta, x, t)$.

The contribution of the middle factor on the right side of (4.3) to the limit of $\varepsilon \log f^{\varepsilon}(k, x, \mathbf{t})$ can be evaluated in two steps. The first step is to express $\tau^{\varepsilon}(i k, x, \mathbf{t})$ given by (4.4) in terms of $\tau^{\varepsilon}(x, t)$ given by (3.9). Upon fixing $\log (z)$ on the sheet where $\log (1)=0$ and with its branch cut on the negative real axis, for every $\eta \in$ $\left[0, \eta_{\max }\right]$ and complex $k$ with $|k|>\eta_{\max }$ one has the convergent expansion

$$
\frac{1}{2} \log \left(\frac{i k-\eta}{i k+\eta}\right)=-\sum_{n=0}^{\infty} \frac{2}{2 n+1}\left(\frac{1}{2 i k}\right)^{2 n+1} 4^{n} \eta^{2 n+1}=\mathrm{s}(i k) \cdot p_{\mathrm{t}}(\eta),
$$

where $p_{t}(\eta)$ is defined after (3.19) and $s(i k)=\left(s_{1}(i k), s_{2}(i k), \ldots\right)$ is defined by

$$
s_{n+1}(i k)=-\frac{2}{2 n+1}\left(\frac{1}{2 i k}\right)^{2 n+1}, \text { for } n=0,1, \ldots
$$

In particular, expansion (4.10) holds over all $k$ in the set $\Omega_{I} \equiv\{k \in \Omega: k$ is imaginary , in which case $s(i k)$ is both real and in $\mathscr{T}$. Hence, by $(4.10)$,

$$
a(\eta, x, \mathbf{t})+\varepsilon \frac{1}{2} \log \left(\frac{i k-\eta}{i k+\eta}\right)=a(\eta, x, \mathbf{t})+\varepsilon \mathbf{s}(i k) \cdot p_{\mathbf{t}}(\eta)=a(\eta, x, \mathbf{t}+\varepsilon \mathbf{s}(i k)) .
$$


This then allows $\tau^{\varepsilon}(i k, x, t)$, given by (4.4), to be expressed as

$$
\tau^{\varepsilon}(i k, x, \mathbf{t})=\tau^{\varepsilon}(x, \mathbf{t}+\varepsilon \mathbf{s}(i k)) .
$$

This identity has played an important role in past work $[2,23,24]$, just as it will here.

The second step is to pass to the limit. The convexity $(3.14)$ of $\log \tau^{\varepsilon}(x, t)$ over $\mathbf{t} \in \mathscr{T}$ provides the basic estimate

$$
\begin{aligned}
\varepsilon^{2} \mathbf{s}(i k) \cdot \partial_{\mathbf{t}} \log \tau^{\varepsilon}(x, \mathbf{t}) & \leqq \varepsilon \log \tau^{\varepsilon}(x, \mathbf{t}+\varepsilon \mathbf{s}(i k))-\varepsilon \log \tau^{\varepsilon}(x, \mathbf{t}) \\
& \leqq \varepsilon^{2} \mathbf{s}(i k) \cdot \partial_{\mathbf{t}} \log \tau^{\varepsilon}(x, \mathbf{t}+\varepsilon \mathbf{s}(i k)) .
\end{aligned}
$$

However, by the $C^{1}$ convergence asserted in (3.20), one has

$$
\lim _{\varepsilon \rightarrow 0} \varepsilon^{2} \partial_{\mathbf{t}} \log \tau^{\varepsilon}(x, \mathbf{t}+\varepsilon \mathbf{s}(i k))=\partial_{\mathbf{t}} q(x, \mathbf{t})=\lim _{\varepsilon \rightarrow 0} \varepsilon^{2} \partial_{\mathbf{t}} \log \tau^{\varepsilon}(x, \mathbf{t}),
$$

where the limits are uniform over compact subsets of $(k, x, \mathbf{t})$ in $\Omega_{I} \times \mathbb{R} \times \mathscr{T}$ and $q(x, t)$ is given by (3.16). Applying this result to (4.14) then using (3.19b) and (4.10) yields the limit

$$
\begin{aligned}
\lim _{\varepsilon \rightarrow 0} \varepsilon \log \left(\frac{\tau^{\varepsilon}(i k, x, \mathbf{t})}{\tau^{\varepsilon}(x, \mathbf{t})}\right)=\mathbf{s}(i k) \cdot \partial_{\mathbf{t}} q(x, \mathbf{t}) & =\frac{2}{\pi} \int_{0}^{\eta_{\max }} \mathbf{s}(i k) \cdot p_{\mathbf{t}}(\eta) \psi^{*}(\eta, x, \mathbf{t}) d \eta, \\
& =\frac{1}{\pi} \int_{0}^{\eta_{\max }} \log \left(\frac{i k-\eta}{i k+\eta}\right) \psi^{*}(\eta, x, \mathbf{t}) d \eta,
\end{aligned}
$$

where again the limits are uniform over compact subsets of $(k, x, t)$ in $\Omega_{I} \times \mathbb{R} \times \mathscr{T}$.

Now turning toward the contribution of the third factor on the right side of (4.3) to the limit of $\varepsilon \log f^{\varepsilon}(k, x, t)$, it follows directly from the definition (3.2) of the $\eta_{j}^{\varepsilon}$ that

$$
\begin{aligned}
\lim _{\varepsilon \rightarrow 0} \varepsilon \log \left(\prod_{j=1}^{N^{\varepsilon}} \frac{i k+\eta_{j}^{\varepsilon}}{i k-\eta_{j}^{\varepsilon}}\right) & =-\frac{1}{\pi} \lim _{\varepsilon \rightarrow 0} \varepsilon \pi \sum_{j=1}^{N^{\varepsilon}} \log \left(\frac{i k-\eta_{j}^{\varepsilon}}{i k+\eta_{j}^{\varepsilon}}\right) \\
& =-\frac{1}{\pi} \int_{0}^{\eta_{\max }} \log \left(\frac{i k-\eta}{i k+\eta}\right) \phi(\eta) d \eta,
\end{aligned}
$$

where $\phi(\eta)$ is the Weyl asymptotic density defined in (3.1).

Finally, by combining (4.16) and (4.17) with (4.3) we establish the limit announced in (1.5) of the introduction, but restricted to those $k$ that are imaginary.

Proposition 4.1. The Jost function $f^{\varepsilon}$ defined in (4.3) satisfies

$$
\begin{aligned}
\lim _{\varepsilon \rightarrow 0} \varepsilon \log f^{\varepsilon}(k, x, \mathbf{t})= & -i k x+p(i k, \mathbf{t}) \\
& -\frac{1}{\pi} \int_{0}^{\eta_{\max }} \log \left(\frac{i k-\eta}{i k+\eta}\right)\left(\phi(\eta)-\psi^{*}(\eta, x, \mathbf{t})\right) d \eta,
\end{aligned}
$$

where the limit is uniform over subsets of $(k, x, \mathbf{t})$ that are compact in $\Omega_{I} \times \mathbb{R} \times \mathscr{T}$. 
The limiting function above extends to an analytic function of $k$ over $\Omega$, all complex $k$ lying outside the imaginary interval [ $\left.-i \eta_{\max }, i \eta_{\max }\right]$. Theorem 5.3 of the next section will show that the limit (4.18) is in fact uniform over subsets of $(k, x, t)$ that are compact in $\Omega \times \mathbb{R} \times \mathscr{T}$.

The limiting form for all the conserved densities and fluxes can now be recovered formally. Comparing weak limits of (4.9) with the appropriate derivatives of (4.18) and passing limits formally through sums yields the relations

$$
\begin{aligned}
\frac{1}{\pi} \int_{0}^{\eta_{\max }} \log \left(\frac{i k-\eta}{i k+\eta}\right) \partial_{x} \psi^{*}(\eta, x, \mathbf{t}) d \eta & =\sum_{\ell=0}^{\infty} \lim _{\varepsilon \rightarrow 0} \rho_{\ell}^{\varepsilon}(x, \mathbf{t})\left(\frac{1}{2 i k}\right)^{\ell+1}, \\
-\frac{1}{\pi} \int_{0}^{\eta_{\max }} \log \left(\frac{i k-\eta}{i k+\eta}\right) \partial_{t_{m}} \psi^{*}(\eta, x, \mathbf{t}) d \eta & =\sum_{\ell=0}^{\infty} \lim _{\varepsilon \rightarrow 0} v_{2 m, \ell}^{\varepsilon}(x, \mathbf{t})\left(\frac{1}{2 i k}\right)^{\ell+1} .
\end{aligned}
$$

Here again, the derivatives on $\psi^{*}$ are to be understood in the sense of distributions. Using the expansion (4.10) of the logarithm on the left side above and comparing coefficients of like powers of $2 i k$ allows one to read off the weak limits

$$
\begin{aligned}
\lim _{\varepsilon \rightarrow 0} \rho_{2 n}^{\varepsilon}(x, \mathbf{t}) & =-\frac{4}{\pi} \frac{4^{n}}{2 n+1} \int_{0}^{\eta_{\max }} \eta^{2 n+1} \partial_{x} \psi^{*}(\eta, x, \mathbf{t}) d \eta, \\
\lim _{\varepsilon \rightarrow 0} v_{2 m, 2 n}^{\varepsilon}(x, \mathbf{t}) & =\frac{4}{\pi} \frac{4^{n}}{2 n+1} \int_{0}^{\eta_{\max }} \eta^{2 n+1} \partial_{t_{m}} \psi^{*}(\eta, x, \mathbf{t}) d \eta,
\end{aligned}
$$

for $n=0,1, \ldots$ from the odd powers of $2 i k$ and

$$
\lim _{\varepsilon \rightarrow 0} \rho_{2 n+1}^{\varepsilon}(x, \mathbf{t})=0, \quad \lim _{\varepsilon \rightarrow 0} v_{2 m, 2 n+1}^{\varepsilon}(x, \mathbf{t})=0,
$$

for $n=0,1, \ldots$ from the even powers. Formulas $(4.20)$ for the weak limits of the conserved densities and fluxes agree with those given earlier in (3.22) and (3.23). This result shows that the maximizer $\psi^{*}$ can be viewed as a generating function that encodes all information about the zero-dispersion limit through relations (4.19).

\section{The Limit of Spectral Measures}

In this section we will extend the validity of the limit described in Proposition 4.1 to all of $\Omega$, the complement of the imaginary interval [ $\left.-i \eta_{\max }, i \eta_{\max }\right]$ in the complex $k$-plane. In the course of doing so we will also interpret $\varepsilon \log f^{\varepsilon}(k, x, t)$ in terms of a spectral measure associated with the Dirichlet eigenvalue problem over half-lines for the Schrödinger operator $\mathscr{L}^{\varepsilon}(t)$ defined in (4.1).

We will begin by recalling the basic ingredients involved in describing solutions of the Dirichlet eigenvalue problem. By the remarks following (4.6) the Jost function $f^{\varepsilon}$ can be written in the form

$$
f^{\varepsilon}(k, x, \mathbf{t})=\exp \left(\frac{-i k x+p(i k, \mathbf{t})}{\varepsilon}\right) \prod_{j=1}^{N^{\varepsilon}} \frac{i k+\xi_{j}^{\varepsilon}(x, \mathbf{t})}{i k-\eta_{j}^{\varepsilon}},
$$

where $k=i \xi_{j}^{\varepsilon}(x, \mathbf{t})$ are the $N^{\varepsilon}$ zeros of the numerator of $\tau^{\varepsilon}(i k, x, t)$. The remarks following (4.8) show that each $\xi_{j}^{\varepsilon}$ lies in the interval $\left[-\eta_{\max }, \eta_{\max }\right]$ and that each nonzero $\mu_{j}^{\varepsilon}(y, \mathbf{t})=-\xi_{j}^{\varepsilon}(y, t)$ is an eigenvalue for the Schrödinger operator $\mathscr{L}^{\varepsilon}(\mathbf{t})$ 
considered over either $L^{2}([y, \infty))$ or $L^{2}((-\infty, y])$ with a Dirichlet boundary condition at $x=y$ depending on whether $\xi_{j}^{\varepsilon}(y, t)$ is negative or positive, respectively. The set of whole-line eigenvalues $\left\{\lambda_{j}^{\varepsilon}\right\}$ is strictly ordered as in (3.5) while classical spectral theory [3] states that those $\left\{\mu_{j}^{\varepsilon}(x, t)\right\}$ that are half-line Dirichlet eigenvalues are simple and can be ordered so as to interlace with the $\left\{\lambda_{j}^{\varepsilon}\right\}$ :

$$
-\eta_{\max }^{2}<\lambda_{1}^{\varepsilon} \leqq \mu_{1}^{\varepsilon}(x, \mathbf{t}) \leqq \cdots \leqq \lambda_{j}^{\varepsilon} \leqq \mu_{j}^{\varepsilon}(x, \mathbf{t}) \leqq \cdots \leqq \lambda_{N^{\varepsilon}}^{\varepsilon} \leqq \mu_{N^{\varepsilon}}^{\varepsilon}(x, \mathbf{t}) \leqq 0,
$$

where equalities may occur at a given $(x, t)$ only as triple coincidences of the form

$$
\cdots<\mu_{j-1}^{\varepsilon}(x, \mathbf{t})=\lambda_{j}^{\varepsilon}=\mu_{j}^{\varepsilon}(x, \mathbf{t})<\cdots .
$$

This convention determines the labeling of the $\left\{\xi_{j}^{\varepsilon}(x, t)\right\}$; however, it should be noted that the ordering of the labels does not correspond to the numerical ordering of the $\left\{\xi_{j}^{\varepsilon}(x, \mathbf{t})\right\}$. The relative ordering (5.2) of the Dirichlet and whole-line eigenvalues with respect to each other will play a crucial role in what follows.

The main result of this section is the following characterization of $\psi^{*}(\eta, x, t)$ in terms of the limiting density of the $\left\{\xi_{j}^{\varepsilon}(x, t)\right\}$ :

Theorem 5.1. In the sense of weak convergence of Borel measures over $\left[0, \eta_{\max }\right]$ one has

$$
\lim _{\varepsilon \rightarrow 0} \varepsilon \pi \sum_{j=1}^{N^{\varepsilon}} \delta\left(\eta+\xi_{j}^{\varepsilon}(x, \mathbf{t})\right) d \eta=\psi^{*}(\eta, x, \mathbf{t}) d \eta,
$$

where the limit is uniform over subsets of $(x, \mathbf{t})$ that are compact in $\mathbb{R} \times \mathscr{T}$.

Only terms that have support in $\left[0, \eta_{\max }\right]$ will contribute to the sum on the left side of (5.3). More specifically, only those terms for which $\xi_{j}^{\varepsilon}(x, t)$ is nonpositive will contribute.

An important ingredient we will use is the notion of a Herglotz function over the domain $\Omega$. A function $h=h(k)$ that is analytic in the domain $\Omega$ will be called Herglotz if it has a representation of the form

$$
h(k)=p(k)+\frac{1}{\pi} \int_{-\eta_{\max }}^{\eta_{\max }} \frac{d \sigma(\eta)}{i k-\eta},
$$

where $p(k)$ is a polynomial that is real-valued when $k$ is imaginary while $d \sigma$ is a nonnegative, finite, regular Borel measure that is supported within the interval $\left[-\eta_{\max }, \eta_{\max }\right]$. It is relatively easy to show that a function that is Herglotz has a unique representation of the form (5.4). This definition differs slightly from that usually given [1] in that we allow a general polynomial behavior and that it is formulated for the domain $\Omega$ rather than for the upper-half plane.

The first result of this section will be to show that

Proposition 5.2. The function $\varepsilon \log f^{\varepsilon}(k, x, t)$ is Herglotz in $\Omega$ with the representation

$$
\varepsilon \log f^{\varepsilon}(k, x, \mathbf{t})=-i k x+p(i k, \mathbf{t})+\frac{1}{\pi} \int_{-\eta_{\max }}^{\eta_{\max }} \frac{d \sigma^{\varepsilon}(\eta ; x, \mathbf{t})}{i k-\eta},
$$

where $d \sigma^{\varepsilon}(\eta ; x, \mathbf{t})=w^{\varepsilon}(\eta ; x, \mathbf{t}) d \eta$ with

$$
w^{\varepsilon}(\eta ; x, \mathbf{t})=\int_{\eta}^{\eta_{\max }} \varepsilon \pi \sum_{j=1}^{N^{\varepsilon}}\left[\delta\left(\xi-\eta_{j}^{\varepsilon}\right)-\delta\left(\xi+\xi_{j}^{\varepsilon}(x, \mathbf{t})\right)\right] d \xi .
$$


Proof. Note that the ordering of the eigenvalues (5.2) ensures that for every $(x, t)$ the $w^{\varepsilon}$ given by (5.5) is nonnegative and vanishes identically for those $\eta$ outside the interval $\left[-\eta_{\max }, \eta_{\max }\right]$. Moreover, it also implies that $w^{\varepsilon}(\eta ; x, t)$ is bounded above uniformly in $(x, t)$ by

$$
\bar{w}^{\varepsilon}(\eta)=\int_{\eta}^{\eta_{\max }} \varepsilon \pi \sum_{j=1}^{N^{\varepsilon}}\left[\delta\left(\xi-\eta_{j}^{\varepsilon}\right)-\delta\left(\xi+\eta_{j}^{\varepsilon}\right)\right] d \xi,
$$

which is bounded above by $\varepsilon \pi N^{\varepsilon}$, which itself is clearly bounded uniformly in $\varepsilon$ by the definition (3.4) of $N^{\varepsilon}$. The associated measure $d \sigma^{\varepsilon}(\eta ; x, t)$ is therefore a nonnegative, finite, regular Borel measure that is supported within the interval $\left[-\eta_{\max }, \eta_{\max }\right]$, so the right side of (5.5) is a representation of the form (5.4). All that remains to be shown is that (5.5) holds.

It can be seen from the representation of $f^{\varepsilon}$ given by (5.1) that

$$
\begin{aligned}
\varepsilon \log f^{\varepsilon}(k, x, \mathbf{t}) & =-i k x+p(i k, \mathbf{t})+\varepsilon \sum_{j=1}^{N^{\varepsilon}}\left[\log \left(i k+\xi_{j}^{\varepsilon}(x, \mathbf{t})\right)-\log \left(i k-\eta_{j}^{\varepsilon}\right)\right] \\
& =-i k x+p(i k, \mathbf{t})+\frac{1}{\pi} \int_{-\eta_{\max }}^{\eta_{\max }} \log (i k-\eta) d w^{\varepsilon}(\eta ; x, \mathbf{t}),
\end{aligned}
$$

where $w^{\varepsilon}(\eta ; x, t)$ is given by (5.6). Integration by parts then yields (5.5), thereby establishing the proposition.

Next, we observe that the Jost function limit given on the right side of (4.18) can be recast in a Herglotz form similar to (5.4). The first step is to extend the functions $\psi^{*}(\cdot, x, t)$ and $\phi(\cdot)$, which are defined over $\left[0, \eta_{\max }\right]$, to the whole real line as odd functions with their support contained within $\left[-\eta_{\max }, \eta_{\max }\right]$. By invoking first this odd symmetry and then integrating by parts, the integral in the last term on the right side of (4.18) can be written as

$$
\begin{aligned}
-\int_{0}^{\eta_{\max }} \log \left(\frac{i k-\eta}{i k+\eta}\right)\left(\phi(\eta)-\psi^{*}(\eta, x, \mathbf{t})\right) d \eta & =\int_{-\eta_{\max }}^{\eta_{\max }} \log (i k-\eta) d w(\eta ; x, \mathbf{t}) \\
& =\int_{-\eta_{\max }}^{\eta_{\max }} \frac{d \sigma(\eta ; x, \mathbf{t})}{i k-\eta},
\end{aligned}
$$

where $d \sigma(\eta ; x, \mathbf{t})=w(\eta ; x, \mathbf{t}) d \eta$ with

$$
w(\eta ; x, \mathbf{t})=\int_{\eta}^{\eta_{\max }}\left[\phi(\xi)-\psi^{*}(\xi, x, \mathbf{t})\right] d \xi .
$$

Hence, the Jost function limit (4.18) becomes

$$
\lim _{\varepsilon \rightarrow 0} \varepsilon \log f^{\varepsilon}(k, x, \mathbf{t})=-i k x+p(i k, \mathbf{t})+\frac{1}{\pi} \int_{-\eta_{\max }}^{\eta_{\max }} \frac{d \sigma(\eta ; x, \mathbf{t})}{i k-\eta},
$$

where the limit is uniform over subsets of $(k, x, t)$ that are compact in $\Omega_{I} \times \mathbb{R} \times \mathscr{T}$. Here again $\Omega_{I}$ denotes the intersection of $\Omega$ with the imaginary axis - in other words, the complement of the interval $\left[-i \eta_{\max }, i \eta_{\max }\right]$ in the imaginary axis. Because, by 
(3.17), $\psi^{*}(\eta, x, t) \leqq \phi(\eta)$ over $\left[0, \eta_{\max }\right]$, for every $(x, t)$ the $w$ given by $(5.10)$ is nonnegative and vanishes identically for those $\eta$ outside the interval $\left[-\eta_{\max }, \eta_{\max }\right]$. Moreover, it also implies that $w^{\varepsilon}(\eta ; x, \mathbf{t})$ is bounded above uniformly in $(x, \mathbf{t})$ by the even extension of $\Phi(\eta)$ over [ $\left.-\eta_{\max }, \eta_{\max }\right]$, where $\Phi(\eta)$ was defined in (3.3) and is bounded above by $\Phi(0)$. The associated measure $d \sigma(\eta ; x, \mathbf{t})$ is therefore a nonnegative, finite, regular Borel measure that is supported within the interval $\left[-\eta_{\max }, \eta_{\max }\right]$, so the right side of (5.11) is a representation of the form (5.4) over $\Omega$.

We can now extend the limit of (4.18) to all of $\Omega$.

Theorem 5.3. The Jost function given by (5.1) satisfies

$$
\lim _{\varepsilon \rightarrow 0} \varepsilon \log f^{\varepsilon}(k, x, \mathbf{t})=-i k x+p(i k, \mathbf{t})+\frac{1}{\pi} \int_{-\eta_{\max }}^{\eta_{\max }} \frac{d \sigma(\eta ; x, \mathbf{t})}{i k-\eta},
$$

uniformly over compact subsets of $\Omega \times \mathbb{R} \times \mathscr{T}$. In particular, the limit is an analytic function in the $k$-plane outside the imaginary interval $\left[-i \eta_{\max }, i \eta_{\max }\right]$.

Proof. By comparing (5.4) with (5.11) one concludes that

$$
\lim _{\varepsilon \rightarrow 0} \int_{-\eta_{\max }}^{\eta_{\max }} \frac{d \sigma^{\varepsilon}(\eta ; x, \mathbf{t})}{i k-\eta}=\int_{-\eta_{\max }}^{\eta_{\max }} \frac{d \sigma(\eta ; x, \mathbf{t})}{i k-\eta}
$$

holds uniformly over compact subsets of $\Omega_{I} \times \mathbb{R} \times \mathscr{T}$. By the Stone-Weierstrass approximation theorem, the linear span of the family of functions $\left\{(i k-\eta)^{-1}: k \in\right.$ $\left.\Omega_{I}\right\}$ is dense in the space of real-valued continuous functions over $\left[-\eta_{\max }, \eta_{\max }\right]$. It follows that for any continuous function $g(\eta)$ one has the limit

$$
\lim _{\varepsilon \rightarrow 0} \int_{-\eta_{\max }}^{\eta_{\max }} g(\eta) d \sigma^{\varepsilon}(\eta ; x, \mathbf{t})=\int_{-\eta_{\max }}^{\eta_{\max }} g(\eta) d \sigma(\eta ; x, \mathbf{t}),
$$

where the limit is uniform over compact subsets of $(g, x, t)$ in $C\left(\left[-\eta_{\max }, \eta_{\max }\right]\right) \times$ $\mathbb{R} \times \mathscr{T}$. The limit (5.13) therefore holds uniformly over compact subsets of $\Omega \times \mathbb{R} \times \mathscr{T}$ because the real and imaginary parts of the integrand will lie in a compact set of $C\left(\left[-\eta_{\max }, \eta_{\max }\right]\right)$ when $k$ lies in a compact subset of $\Omega$. The theorem then follows by passing to the limit in (5.5).

The convergence of $d \sigma^{\varepsilon}$ to $d \sigma$ indicated in (5.14) is that of the weak convergence of measures. This convergence can be strengthened through the following result concerning the convergence of their densities, $w^{\varepsilon}$ to $w$, defined by (5.6) and (5.10).

Proposition 5.4. The measures $d w^{\varepsilon}$ and $d w$ defined by

$$
\begin{aligned}
d w^{\varepsilon}(\eta ; x, \mathbf{t}) & =-\varepsilon \pi \sum_{j=1}^{N^{\varepsilon}}\left[\delta\left(\eta-\eta_{j}^{\varepsilon}\right)-\delta\left(\eta+\xi_{j}^{\varepsilon}(x, \mathbf{t})\right)\right] d \eta \\
d w(\eta ; x, \mathbf{t}) & =-\left[\phi(\eta)-\psi^{*}(\eta, x, \mathbf{t})\right] d \eta
\end{aligned}
$$

satisfy

$$
\lim _{\varepsilon \rightarrow 0} d w^{\varepsilon}(\eta ; x, \mathbf{t})=d w(\eta ; x, \mathbf{t})
$$


where the convergence is in the weak topology of Borel measures over $\left[-\eta_{\max }, \eta_{\max }\right]$ and is uniform over compact subsets of $(x, \mathbf{t})$ in $\mathbb{R} \times \mathscr{T}$.

Remark. Theorem 5.1 follows immediately from this proposition upon restricting the measures in (5.16) to $\left[0, \eta_{\max }\right]$ and using the consequence of definition (3.2) that $\phi(\eta)$ is the limiting density of the $\eta_{j}^{\varepsilon}$.

Proof. For every $g$ in $C\left(\left[-\eta_{\max }, \eta_{\max }\right]\right)$ we must establish

$$
\lim _{\varepsilon \rightarrow 0} \int_{-\eta_{\max }}^{\eta_{\max }} g(\eta) d w^{\varepsilon}(\eta ; x, \mathbf{t})=\int_{-\eta_{\max }}^{\eta_{\max }} g(\eta) d w(\eta ; x, \mathbf{t}),
$$

where the limit is uniform over compact subsets of $(x, t)$ in $\mathbb{R} \times \mathscr{T}$. However, it is clear from (5.15) that the total variation of the $d w^{\varepsilon}(\cdot ; x, \mathbf{t})$ satisfies the bound

$$
\left\|d w^{\varepsilon}(\cdot ; x, \mathbf{t})\right\|_{T V} \leqq \int_{-\eta_{\max }}^{\eta_{\max }} \varepsilon \pi \sum_{j=1}^{N^{\varepsilon}}\left[\delta\left(\eta-\eta_{j}^{\varepsilon}\right)+\delta\left(\eta+\xi_{j}^{\varepsilon}(x, \mathbf{t})\right)\right] d \eta=2 \varepsilon \pi N^{\varepsilon},
$$

which, by the definition (3.4) of $N^{\varepsilon}$, is uniform in $\varepsilon$ and $(x, \mathbf{t})$. Hence, by a standard density argument, it suffices to establish (5.17) for an arbitrary $g$ in $C^{1}\left(\left[-\eta_{\max }, \eta_{\max }\right]\right)$. For such a $g$ integration by parts yields

$$
\begin{aligned}
& \int_{-\eta_{\max }}^{\eta_{\max }} g(\eta) d w^{\varepsilon}(\eta ; x, \mathbf{t})=-\int_{-\eta_{\max }}^{\eta_{\max }} w^{\varepsilon}(\eta ; x, \mathbf{t}) g^{\prime}(\eta) d \eta, \\
& \int_{-\eta_{\max }}^{\eta_{\max }} g(\eta) d w(\eta ; x, \mathbf{t})=-\int_{-\eta_{\max }}^{\eta_{\max }} w(\eta ; x, \mathbf{t}) g^{\prime}(\eta) d \eta .
\end{aligned}
$$

The convergence (5.14) implies that

$$
\lim _{\varepsilon \rightarrow 0} \int_{-\eta_{\max }}^{\eta_{\max }} w^{\varepsilon}(\eta ; x, \mathbf{t}) g^{\prime}(\eta) d \eta=\int_{-\eta_{\max }}^{\eta_{\max }} w(\eta ; x, \mathbf{t}) g^{\prime}(\eta) d \eta,
$$

where the limit is uniform over compact subsets of $(x, \mathbf{t})$ in $\mathbb{R} \times \mathscr{T}$. By (5.19), this establishes (5.17) for every $g$ in $C^{1}\left(\left[-\eta_{\max }, \eta_{\max }\right]\right)$. As was argued from (5.18), the proposition then follows.

\section{The Behavior of the Weyl Function}

At the end of Sect. 4 it was observed that Proposition 4.18 enables one to recover the limiting form of the conserved densities and fluxes, at least in terms of distributional derivatives of $\psi^{*}$. In this section we show that Theorem 5.1 allows us to strengthen this characterization of the limit of the densities. Precisely, this limit is a measure in $x$ and the limit holds in the sense of weak convergence of measures in $x$.

This analysis is mediated by the scaled form of the Weyl function introduced in (2.8), which, when $f^{\varepsilon}$ is given by (5.1), becomes

$$
m^{\varepsilon}(k, x, \mathbf{t}) \equiv \varepsilon \partial_{x} \log f^{\varepsilon}(k, x, \mathbf{t})=-i k+\varepsilon \sum_{j=1}^{N^{\varepsilon}} \frac{\partial_{x} \xi_{j}^{\varepsilon}(x, \mathbf{t})}{i k+\xi_{j}^{\varepsilon}(x, \mathbf{t})} .
$$

We will first give a self-contained argument that $m^{\varepsilon}$ is Herglotz in $\Omega$ and, in so doing, construct the associated Weyl spectral measure $d \sigma_{x}^{\varepsilon}$. We will then consider the zero-dispersion limit of this measure which carries the limiting form of the conserved densities. 
Proposition 6.1. The function $m^{\varepsilon}(k, x, t)$ is Herglotz in $\Omega$ with the representation

$$
m^{\varepsilon}(k, x, \mathbf{t})=-i k+\frac{1}{\pi} \int_{-\eta_{\max }}^{\eta_{\max }} \frac{d \sigma_{x}^{\varepsilon}(\eta ; x, \mathbf{t})}{i k-\eta},
$$

where $d \sigma_{x}^{\varepsilon}(\eta ; x, \mathbf{t})=\partial_{x} w^{\varepsilon}(\eta ; x, \mathbf{t}) d \eta$ with

$$
\partial_{x} w^{\varepsilon}(\eta ; x, \mathbf{t})=\varepsilon \pi \sum_{j=1}^{N^{\varepsilon}} \partial_{x} \xi_{j}^{\varepsilon}(x, \mathbf{t}) \delta\left(\eta+\xi_{j}^{\varepsilon}(x, \mathbf{t})\right) .
$$

Note that formulas (6.2) and (6.3) follow directly from (6.1), so all that needs to be established is that $d \sigma_{x}^{\varepsilon}(\eta ; x, t)$ is a nonnegative, finite Borel measure. By (6.3), all of these properties will follow from the next lemma.

\section{Lemma 6.2.}

$$
\varepsilon \partial_{x} \xi_{j}^{\varepsilon}(x, \mathbf{t})=\left(\mu_{j}^{\varepsilon}(x, \mathbf{t})-\lambda_{j}^{\varepsilon}\right) \prod_{\substack{l=1 \\ l \neq j}}^{N^{\varepsilon}} \frac{\mu_{j}^{\varepsilon}(x, \mathbf{t})-\lambda_{l}^{\varepsilon}}{\mu_{j}^{\varepsilon}(x, \mathbf{t})-\mu_{l}^{\varepsilon}(x, \mathbf{t})} \geqq 0 .
$$

Proof. The nonnegativity of the expression to the right of the equality in (6.4) follows directly from the comparison inequalities (5.2), which show that the prefactor and each quotient in the product is nonnegative. Therefore, the lemma will follow upon establishing the equality in (6.4).

This identity is ascertained by comparing two different expressions for $m_{o}^{\varepsilon}(k, x, t)$, the odd component of $m^{\varepsilon}$ as a function of $k$ which was introduced in Sect. 2. The first expression for $m_{o}^{\varepsilon}(k, x, t)$ follows directly from taking the odd component of (6.1), namely

$$
m_{o}^{\varepsilon}(k, x, \mathbf{t})=-i k\left(1+\varepsilon \sum_{j=1}^{N^{\varepsilon}} \frac{\partial_{x} \xi_{j}^{\varepsilon}(x, \mathbf{t})}{k^{2}-\mu_{j}^{\varepsilon}(x, \mathbf{t})}\right),
$$

where once again $\mu_{j}^{\varepsilon}(x, t)=-\xi_{j}^{\varepsilon}(x, t)$. The second expression for $m_{o}^{\varepsilon}(k, x, t)$ derives from the fact that $f_{+}^{\varepsilon}=f^{\varepsilon}(k, x, t)$ and $f_{-}^{\varepsilon}=f^{\varepsilon}(-k, x, t)$ are solutions of the Schrödinger equation (4.1) whose Wronskian can be evaluated using the large $x$ asymptotics of $f^{\varepsilon}$ given in (4.7) to obtain

$$
\mathscr{W}\left(f_{+}^{\varepsilon}, f_{-}^{\varepsilon}\right) \equiv f^{\varepsilon}(k, x, \mathbf{t}) \partial_{x} f^{\varepsilon}(-k, x, \mathbf{t})-f^{\varepsilon}(-k, x, \mathbf{t}) \partial_{x} f^{\varepsilon}(k, x, \mathbf{t})=\frac{2 i k}{\varepsilon} .
$$

It follows from (6.6) and (5.1) that

$$
\begin{aligned}
m_{o}^{\varepsilon}(k, x, \mathbf{t}) & =\frac{\varepsilon}{2} \partial_{x} \log \left(\frac{f^{\varepsilon}(k, x, \mathbf{t})}{f^{\varepsilon}(-k, x, \mathbf{t})}\right) \\
& =-\frac{\varepsilon}{2} \frac{\mathscr{W}\left(f_{+}^{\varepsilon}, f_{-}^{\varepsilon}\right)}{f^{\varepsilon}(k, x, \mathbf{t}) f^{\varepsilon}(-k, x, \mathbf{t})}=-i k \prod_{j=1}^{N^{\varepsilon}} \frac{k^{2}-\lambda_{j}^{\varepsilon}}{k^{2}-\mu_{j}^{\varepsilon}(x, \mathbf{t})},
\end{aligned}
$$

where once again $\lambda_{j}^{\varepsilon}=-\eta_{j}^{\varepsilon 2}$. The equality in (6.4) is established by equating the residues of $m_{o}^{\varepsilon}(k, x, t)$ in expressions (6.5) and (6.7), thus completing the proofs of both Lemma 6.2 and Proposition 6.1. Hence, (6.2) gives a representation of $m^{\varepsilon}(k, x, t)$ in the form (5.4) and (6.3) gives the associated Weyl spectral measure $d \sigma_{x}^{\varepsilon}(\eta ; x, \mathbf{t})$. 
What can be said now about the zero-dispersion limit of the measures $d \sigma_{x}^{\varepsilon}$ ? We have the following ingredients for the potential description of this limit.

As a consequence of Theorem 5.1, for any continuous $g$ one has

$$
\lim _{\varepsilon \rightarrow 0} \varepsilon \pi \int_{-\eta_{\max }}^{\eta_{\max }} g(\eta) \sum_{j=1}^{N^{\varepsilon}} \theta\left(-\eta-\xi_{j}^{\varepsilon}(x, \mathbf{t})\right) d \eta=\int_{-\eta_{\max }}^{\eta_{\max }} g(\eta) \int_{\eta}^{\eta_{\max }} \psi^{*}(\xi, x, \mathbf{t}) d \xi d \eta,
$$

where the limit is uniform over compact subsets of $(x, t)$ in $\mathbb{R} \times \mathscr{T}$, and $\theta$ is the Heaviside function. Then for any compactly supported differentiable function $h=h(x)$ one has

$$
\begin{gathered}
\lim _{\varepsilon \rightarrow 0} \int_{-\infty}^{\infty} h^{\prime}(x) \int_{-\eta_{\max }}^{\eta_{\max }} g(\eta) \varepsilon \pi \sum_{j=1}^{N^{\varepsilon}} \theta\left(-\eta-\xi_{j}^{\varepsilon}(x, \mathbf{t})\right) d \eta d x \\
=\int_{-\infty}^{\infty} h^{\prime}(x) \int_{-\eta_{\max }}^{\eta_{\max }} g(\eta) \int_{\eta}^{\eta_{\max }} \psi^{*}(\xi, x, \mathbf{t}) d \xi d \eta d x,
\end{gathered}
$$

where one still has uniformity of the limit over subsets of $t$ that are compact in $\mathscr{T}$. Integration by parts in the terms on the left side of (6.9) gives

$$
\int_{-\infty}^{\infty} h^{\prime}(x) \int_{-\eta_{\max }}^{\eta_{\max }} g(\eta) \varepsilon \pi \sum_{j=1}^{N^{\varepsilon}} \theta\left(-\eta-\xi_{j}^{\varepsilon}(x, \mathbf{t})\right) d \eta d x=\int_{-\infty}^{\infty} h(x) d \gamma^{\varepsilon}(x, \mathbf{t}),
$$

where the $x$-measure $d \gamma^{\varepsilon}(\cdot, t)$ is defined by

$$
d \gamma^{\varepsilon}(x, \mathbf{t}) \equiv \varepsilon \pi \sum_{j=1}^{N^{\varepsilon}} g\left(-\xi_{j}^{\varepsilon}(x, \mathbf{t})\right) \partial_{x} \xi_{j}^{\varepsilon}(x, \mathbf{t}) d x
$$

The total variation of $d \gamma^{\varepsilon}(\cdot, t)$ is bounded by

$$
\left\|d \gamma^{\varepsilon}(\cdot, \mathbf{t})\right\|_{T V} \leqq\|g\|_{\text {sup }} \varepsilon \pi N^{\varepsilon},
$$

which is uniformly bounded in both $\varepsilon$ and $\mathbf{t}$. Hence, the $d \gamma^{\varepsilon}(\cdot, \mathbf{t})$ lie in a set that is compact in the weak topology of Borel measures. Hence, there exists a Borel measure $d \gamma(\cdot, \mathbf{t})$ such that

$$
\begin{aligned}
\int_{-\infty}^{\infty} h(x) d \gamma(x, \mathbf{t}) & =\lim _{\varepsilon \rightarrow 0} \int_{-\infty}^{\infty} h(x) d \gamma^{\varepsilon}(x, \mathbf{t}) \\
& =\int_{-\infty}^{\infty} h^{\prime}(x) \int_{-\eta_{\max }}^{\eta_{\max }} g(\eta) \int_{\eta}^{\eta_{\max }} \psi^{*}(\xi, x, \mathbf{t}) d \xi d \eta d x .
\end{aligned}
$$

From this we read off that

$$
d \gamma(x, \mathbf{t})=-\int_{-\eta_{\max }}^{\eta_{\max }} g(\eta) \int_{\eta}^{\eta_{\max }} \partial_{x} \psi^{*}(\xi, x, \mathbf{t}) d \xi d \eta d x
$$


This yields the following stronger version of the first formula in (4.19):

Theorem 6.3. As densities of the Lebesgue measure $d x$,

$$
\begin{aligned}
\lim _{\varepsilon \rightarrow 0} \varepsilon \partial_{x} \log f^{\varepsilon}(k, x, \mathbf{t}) & =-i k+\lim _{\varepsilon \rightarrow 0} \sum_{\ell=0}^{\infty} \rho_{\ell}^{\varepsilon}(x, \mathbf{t})\left(\frac{1}{2 i k}\right)^{\ell+1} \\
& =-i k+\lim _{\varepsilon \rightarrow 0} \varepsilon \sum_{j=1}^{N^{\varepsilon}} \frac{\partial_{x} \xi_{j}^{\varepsilon}(x, \mathbf{t})}{i k+\xi_{j}^{\varepsilon}(x, \mathbf{t})} \\
& =-i k-\frac{1}{\pi} \int_{-\eta_{\max }}^{\eta_{\max }} \partial_{x}\left[\int_{\eta}^{\eta_{\max }} \frac{\psi^{*}(\xi, x, \mathbf{t})}{i k-\eta} d \xi\right] d \eta,
\end{aligned}
$$

where the limit is uniform over subsets of $(k, t)$ that are compact in $\Omega \times \mathscr{T}$.

In particular, this limit holds in the sense of weak convergence of Borel measures in $x$; i.e., the first equality of (6.13) holds with $h(x)$ any compactly supported measurable function of $x$. This follows because differentiable functions are dense in the class of measurable functions on compact sets. As a consequence it follows that the integral

$$
\int_{\eta}^{\eta_{\max }} \frac{\psi^{*}(\xi, x, \mathbf{t})}{i k-\eta} d \xi
$$

has a measure-valued $x$-derivative.

One expects to have a similar improvement of the second formula in (4.19). In fact, in a subsequent paper [6], we develop estimates on $\partial_{t_{m}} \xi_{j}$ which enable us to control the limiting form of the fluxes in a similar manner.

\section{Comments and Conclusions}

In this paper we have given a complete description of a singular limit (the so-called dispersive limit) of the logarithm of the Jost functions, in terms of $k$-analyticity properties, for the class of operators described by (1.3) (Theorem 5.1 and Theorem 5.3). We have also given a precise statement of what we can say about the limit of the spatial derivatives of these functions. Once again we may state $k$-analyticity properties but for functions with values in $x$-measures (Theorem 6.3). However, it is known $[14,15]$ that the densities converge not in the weak topology of measures, but in the weak- $L^{1}$ topology. We therefore feel that the convergence in Theorem 6.3 can be strengthened accordingly.

Getting better control on the densities would seem to require a better understanding of the mechanism for the formation of singularities in the evolution of the weak limit or the emergence of phase transitions in this limit at critical times t. We know [19] that at those values of $(x, \mathbf{t})$ where the zero-dispersion limit is weak, open gaps exist in the support of $\partial_{x} \psi^{*}(\cdot, x, t)$, which generally consists of a finite number of bands within the interval $\left[0, \eta_{\max }\right]$ such as shown in Fig. 7.1. Numerical studies of the solution, for small $\varepsilon$, at such times when the number of bands is greater than one, show the formation of modulated oscillations [20].

These bands may fission or fuse as $t$ changes but there is not a good understanding of this process. Except in extremely special cases (such as monotone initial 


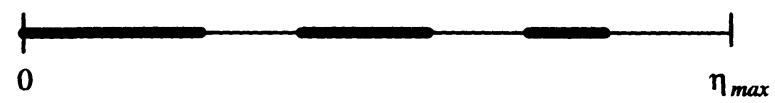

Fig. 7.1 Typically the support of $\partial_{x} \psi^{*}(\cdot, x, t)$ for some value of $(x, t)$ is a finite number of bands within the interval $\left[0, \eta_{\max }\right]$ as depicted in bold above

data [4]) one cannot predict this evolution of band structure as a function of initial data. Our results show that the bands are the regions of support for the limit of the $d \sigma^{\varepsilon}$, where here by "support" we mean intervals in $\eta$ over which the limiting measure is independent of $x$. This makes it clear that the band structure is a consequence of the limiting $x$-dynamics of the $\xi_{j}$ and changes in the band structure are determined by how this dynamics changes as $\mathbf{t}$ is varied. So one natural line of inquiry would be to systematically investigate the $x-t$ dynamics of the $\xi_{j}$ for small values of $\varepsilon$. It will be especially important to determine what aspects of this dynamics triggers the spontaneous emergence of oscillations which are the signature of the phase transitions.

Finally, we expect similar results to hold for any class of initial data for which the $\mathrm{KdV}$ equation is now known to be completely integrable. The same story should be true for the semiclassical limit of the defocusing nonlinear Schrödinger equation, where structures similar to those for $\mathrm{KdV}$ exist [13-15]. It is less obvious, but likely that similar results also hold for the semiclassical limit of the odd flows in the focusing NLS hierarchy with real-valued data, a case that includes the modified KdV equation while excluding the NLS equation itself. This limit was established in [5] even though the associated spectral problem is not self-adjoint, a structural difference that makes the generalization of the present results to this case interesting.

Acknowledgements. We thank Hermann Flaschka for his interest and encouragement in this project and for helpful suggestions. We also thank Percy Deift for his very helpful suggestions.

The authors gratefully acknowledge the support provided to them: N.M.E. from the NSF under grant DMS-8942390 and from the Air Force under grant AFOSR-FQ8671-9000589; C.D.L. from the Air Force under grant AFOSR-90-021 and from the NSF under grant DMS-9404570. In addition, some of this work was carried out while the first two authors were visiting the Mathematical Sciences Research Institute (MSRI) in Berkeley, which is supported in part by the NSF under grant DMS-9022140.

\section{References}

1. Carmona, R. and Lacroix, J.: Spectral Theory of Random Schrödinger Operators. Boston: Birkhäuser 1990

2. Date, E., Jimbo, M., Kashiwara, M. and Miwa, T.: Transformation Groups for Soliton Equations. Proceedings of R.I.M.S. Symposium on "Nonlinear Integrable Systems-Classical Theory and Quantum Theory," Singapore, World Scientific 1983

3. Deift, P. and Trubowitz, E.: Inverse Scattering on the Line. Comm. Pure \& Appl. Math. 32, 121-251 (1979)

4. Ercolani, N., Gabitov, I., Levermore, C.D. and Serre, D., eds.: Singular Limits of Dispersive Waves, Proceedings of the NATO Advanced Research Workshop, 8-12 July 1991, Lyon, NATO ARW Series B 320, New York: Plenum 1994

5. Ercolani, N., Jin, S., Levermore, C.D. and MacEvoy, W.: The Zero Dispersion Limit of the NLS/mKdV Hierarchy for the Nonselfadjoint ZS Operator. Preprint (1993) 
6. Ercolani, N., Levermore, C.D. and Zhang, T.: Weyl Functions via Dirichlet Spectrum and the KdV Zero Dispersion Limit. Preprint (1996)

7. Faddeev, L.: Inverse Problem in Quantum Theory of Scattering. J. Math. Phys. 4, 72-104 (1963)

8. Flaschka, H.: Construction of Conservation Laws for Lax Equations: Comments on a Paper by G. Wilson. Quart. J. Math. Oxford 34, 61-65 (1983)

9. Flaschka, H., Forest, M.G. and McLaughlin, D.W.: Multiphase Averaging and the Inverse Spectral Solutions of the Korteweg-de Vries Equation. Comm. Pure \& Appl. Math. 33, 739784 (1980)

10. Gardner, C.S.: The Korteweg-de Vries Equation and Generalizations IV: The Kortewegde Vries Equation as a Hamiltonian System. J. Math. Phys. 12, 1548-1551 (1971)

11. Gardner, C.S., Greene, J.M., Kruskal, M.D. and Miura, R.M.: Method for Solving the Korteweg-deVries Equation. Phys. Rev. Lett. 19, 1095-1097 (1967)

12. Gardner, C.S., Greene, J.M., Kruskal, M.D. and Miura, R.M.: The Korteweg-deVries Equation and Generalizations VI: Methods for Exact Solutions. Comm. Pure \& Appl. Math. 27, 97-133 (1974)

13: Jin, S.: The Semiclassical Limit of the Defocusing Nonlinear Schrödinger Flows. Ph.D. Dissertation, University of Arizona (1991)

14. Jin, S., Levermore, C.D. and McLaughlin, D.W.: The Behavior of Solutions of the NLS Equation in the Semiclassical Limit. In: "Singular Limits of Dispersive Waves," N. Ercolani, I. Gabitov, C.D. Levermore and D. Serre eds., NATO ARW Series B 320, New York: Plenum 1994, pp. 235-255

15. Jin, S., Levermore, C.D. and McLaughlin, D.W.: The Semiclassical Limit of the Defocusing NLS Hierarchy. Comm. Pure \& Appl. Math. (submitted 1996)

16. Kay, I. and Moses, H.E.: Reflectionless Transmission through Dielectrics and Scattering Potentials. J. Appl. Phys. 27, 1503-1508 (1956)

17. Lax, P.D.: Integrals of Nonlinear Equations of Evolution and Solitary Waves. Comm. Pure \& Appl. Math. 21, 467-490 (1968)

18. Lax, P.D. and Levermore, C.D.: The Zero Dispersion Limit of the Korteweg-deVries Equation. Proc. Nat. Acad. Sci. USA 76, 3602-3606 (1979)

19. Lax, P.D. and Levermore, C.D.: The Small Dispersion Limit of the Korteweg-deVries Equation I, II, III. Comm. Pure \& Appl. Math. 36, 253-290, 571-593, 809-829 (1983)

20. Lax, P.D., Levermore, C.D. and Venakides, S.: The Generation and Propagation of Oscillations in Dispersive IVPs and Their Limiting Behavior. In: "Important Developments in Soliton Theory 1980-1990," T. Fokas and V.E. Zakharov eds., New York: Springer-Verlag, 1993, pp. 205-241.

21. Levermore, C.D.: The Hyperbolic Nature of the Zero Dispersion KdV Limit. Comm. P.D.E. 13, 495-514 (1988)

22. Newell, A.C.: Solitons in Mathematics and Physics. CBMS-NSF Regional Conference Series in Applied Matematics 48, Philadelphia PA: SIAM 1985

23. Sato, M. and Sato, Y.: Soliton Equations as Dynamical Systems on Infinite Dimensional Grassmann Manifolds. In the Proceedings of "Nonlinear Partial Differential Equations in Applied Science (Tokyo 1982)," North-Holland, Math. Stud. 81, 259-271 (1983)

24. Segal, G. and Wilson, G.: Loop Groups and Equations of KdV Type. I.H.E.S. Publ. Math. 61, 5-65 (1985)

25. Venakides, S.: The Zero Dispersion Limit of the Korteweg-deVries Equation with Nontrivial Reflection Coefficient. Comm. Pure \& Appl. Math. 38, 125-155 (1985)

26. Venakides, S.: The Generation of Modulated Wavetrains in the Solution of the KortewegdeVries Equation. Comm. Pure \& Appl. Math. 38, 883-909 (1985)

27. Venakides, S.: The Zero Dispersion Limit of the Korteweg-deVries Equation with Periodic Initial Data. AMS Trans. 301, 189-225 (1987)

28. Venakides, S.: Higher Order Lax-Levermore Theory. Comm. Pure \& Appl. Math. 43, 335-362 (1990)

29. Whitham, G.B.: Non-Linear Dispersive Waves. Proc. Royal Soc. London Ser. A 283, 238-261 (1965)

30. Whitham, G.B.: Linear and Nonlinear Waves. New York: J. Wiley 1974

31. Wilson, G.: On Two Constructions of Conservation Laws for Lax Equations. Quart. J. Math. Oxford 32, 491-512 (1981) 
32. Zakharov, V.E. and Faddeev, L.: The Korteweg-de Vries Equation: A Completely Integrable Hamiltonian System. Funct. Anal. Appl. 5, 280-287 (1971)

Communicated by M. Jimbo 
